\title{
SN 1054, SN 1006, \& Nova WZ Sagittae Calving Antarctic Ice Cap-Global Warming \& Moose Die Off Longitude Relationship Deflection Area in Antarctica and USA-WZ Sagittae Impact and Martian Dust Storms
}

\author{
William P. Sokeland \\ Spacecraft and Turbine Engines, Oakland City, Indiana 47660, USA
}

\begin{abstract}
The SNIT theory proposes the effects of exploding stars, supernova and nova, on the biosphere of Earth. The feature on our planet that is most sensitive to the incoming energy of an exploding star is sea ice at the poles and this brings in the concept of global warming. The incoming particle streams for SN 1054, SN 1006, and Nova WZ Sagittae have left easily interpreted data noted as calving under the Antarctic ice cap that matches previously predicted longitude locations for the local heat input of the particle streams of the exploding stars. Animal die offs are also caused by the incoming particle streams. The deflection area longitude range for SN 1054 calving produces the correct longitude range for the moose die off beginning in the northern USA in 2006. Some Martian dust storms are created by the wave front of the exploding star debris streams. The explanation of these exploding stars effects using the SNIT theory is the object of this work.
\end{abstract}

Key words: Warming, global, supernova, dust, calving, Antarctica, moose, Martian.

\section{Introduction}

The data and most figures in the six papers of the SNIT theory were found by surfing the internet because the author is handicapped and does not have access to major university libraries. The author wishes to thank the creators of the data and figures cited in his publications because the SNIT theory would not have evolved over the past 20 years without their input.

The first step in the SNIT analysis was to find the arrival times or Estimated Time of Arrivals, ETAs, of nova or supernova debris streams as they impacted our planet. This was successfully done and displayed by the equations for ETA in the Introduction section, and the fit of the arrival times generated for the ice data by the first three red arrows in Fig. 3a of Ref. [1] and the

Corresponding author: William P. Sokeland, MSc, scientist, research fields: turbine engines, spacecraft, comets, tornadoes, hurricanes, and earth thermal conditions.
SN G109.1-1.0 and SN Vela impact times of 15,000 and 11,700 years ago, respectively, in Fig. 7 of Ref. [1].

The next step was to locate the Longitudes, L, of the western and eastern termini, WT and ET, and Cosmic Accumulation of Meteors, CAM, date by the equations in the Introduction section of Ref. [1] pg 136. The derivations of the equations are simple, but the assumption used to locate the termini longitudes is in Ref. [3] section 16 pgs 213-215.

\section{Discussion}

The terms used to describe the SNIT theory can be found in previously published references that are found in open access magazines and Academia.edu [1-5]. Refs. [4] and [5] are best displayed by going to Academia.edu and searching on the references' titles. The reading of Ref. [1] is highly recommended for the understanding of the terms used in the discussion of the SNIT theory. 


\section{Calving}

An excellent piece of data produced by Depoorter M. A., et al. is shown in Fig. 1.

The rate of melting of ice shelves from the bottom of the ice where they rests on the Antarctic continent is displayed in Fig. 1 by various colors. The red, orange, and yellow on the western shore of Antarctica show high melt rates by calving and are attributed to volcanoes that are under the ice. The red line in Fig. 1 connects the western terminus longitude, $155^{\circ} \mathrm{W}$, to the eastern terminus longitude, $25^{\circ} \mathrm{E}$, for SN 1054 whose debris stream began impacting Earth near the year 2006 [2, 3]. The southern tine of Satan's pitchfork created by the incoming particle debris stream of SN 1054 causes all incoming thermal energy that melts ice from the supernova to be deposited in the Antarctic ice to the left of the red line or in the near vicinity of the western or eastern termini of the line [3]. The blue regions specified by the green stars show melt areas associated with the western and eastern termini and also the deflection area, DA, of SN 1054's debris streams. The CAM date for the western terminus, WT, and deflection area is December 12 and represents the day of incoming maximum particle density for those calving areas.

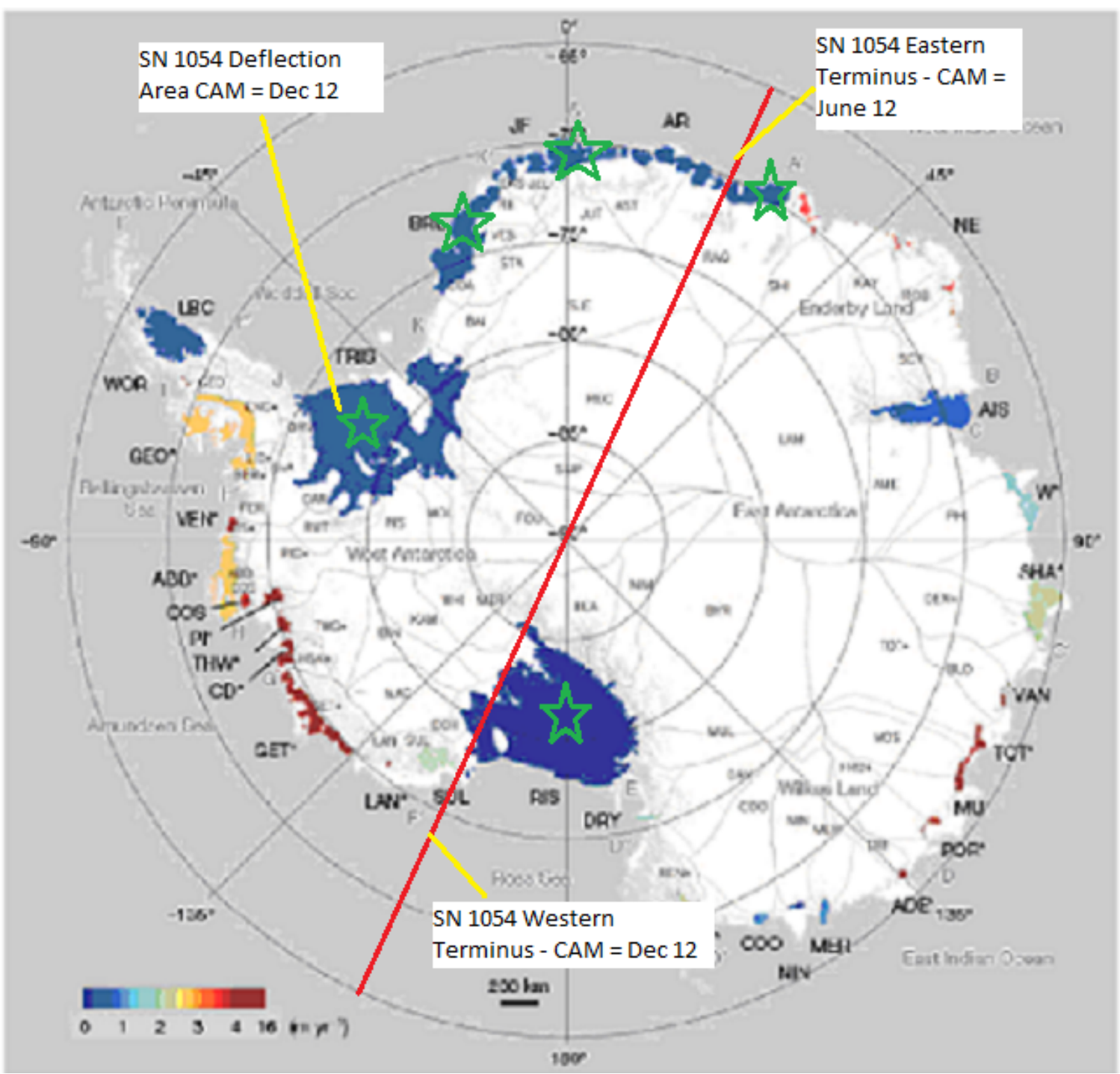

Fig. 1 Mean basal mass-loss rates of Antarctic ice shelves SN 1054 \& 1006 features [6]. 
The particle streams of the western terminus and the associated area of deflection shown in Fig. 1 are caused to move south and north, annually, by the sun's changing magnetic field being located between the locations of the supernova remnant and our planet. The CAM dates are specified for the blue areas noted by the green stars. The maximum deposit of energy by the western terminus and the deflection area particle streams of SN 1054 occur on the CAM date of December 12.

\section{SN 1006 Future Calving}

The green line in Fig 2 connects the western terminus longitude, $13^{\circ} \mathrm{W}$, to the eastern terminus, ET, longitude, $167^{\circ} \mathrm{E}$, for SN 1006 whose debris stream impacted Earth near the year 2012. SN 1006 is not displaying basal mass-loss in Fig. 2 because the southern tine of the younger supernova, SN 1006, impact has not moved far enough south at the time the data were measured for Fig. 2. The basal mass-loss in the western circle may be the exception if the calving data in the circle were taken between 2014 and 2016.

In Fig. 3, there is a sudden dip to the negative for the Antarctic sea ice anomaly for the years 2005 and 2006. This decrease in sea ice area can be attributed to the SN 1054 debris stream encroaching on the sea ice

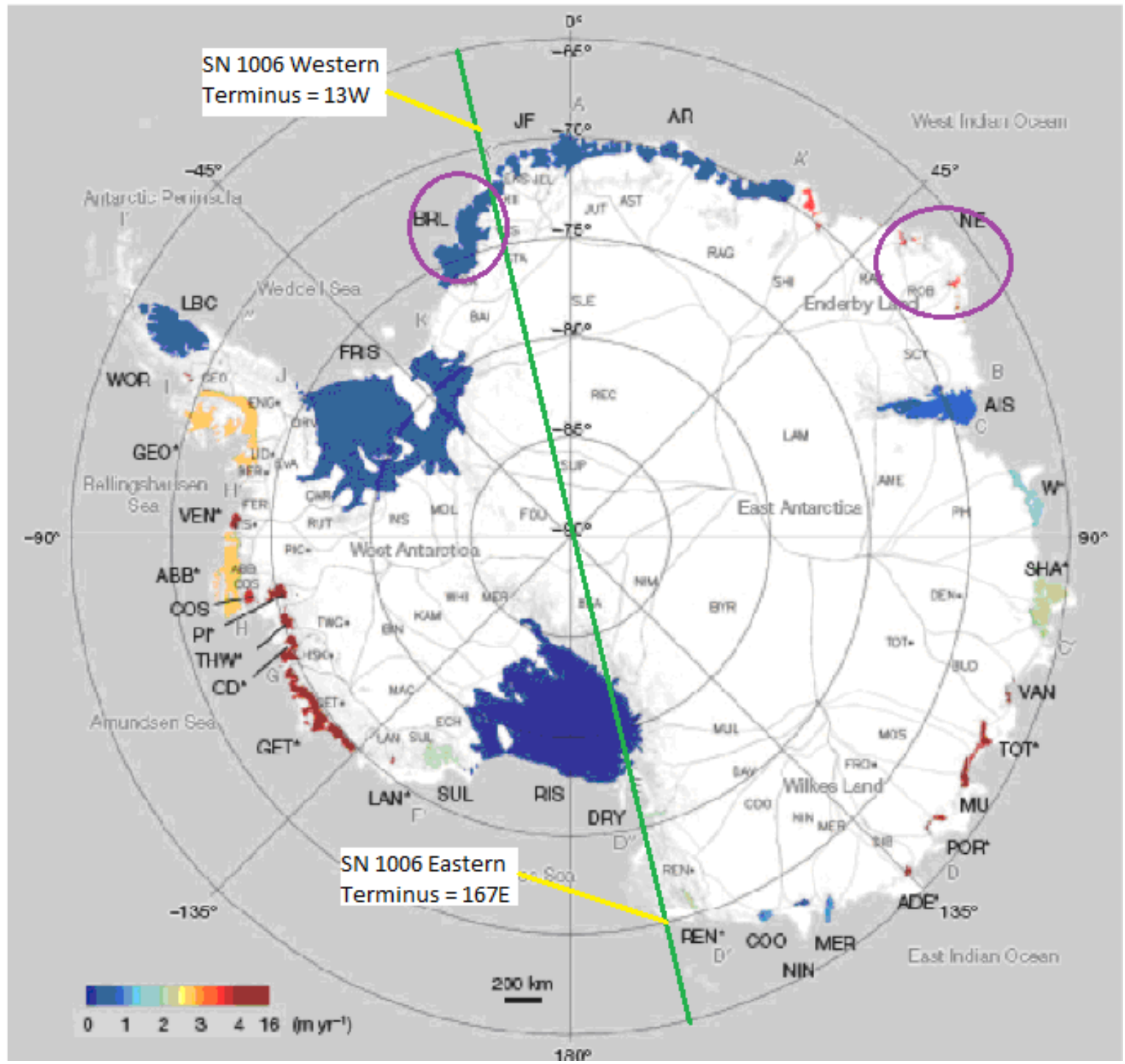

Fig. 2 Future calving for SN 1006 noted purple circles [6]. 


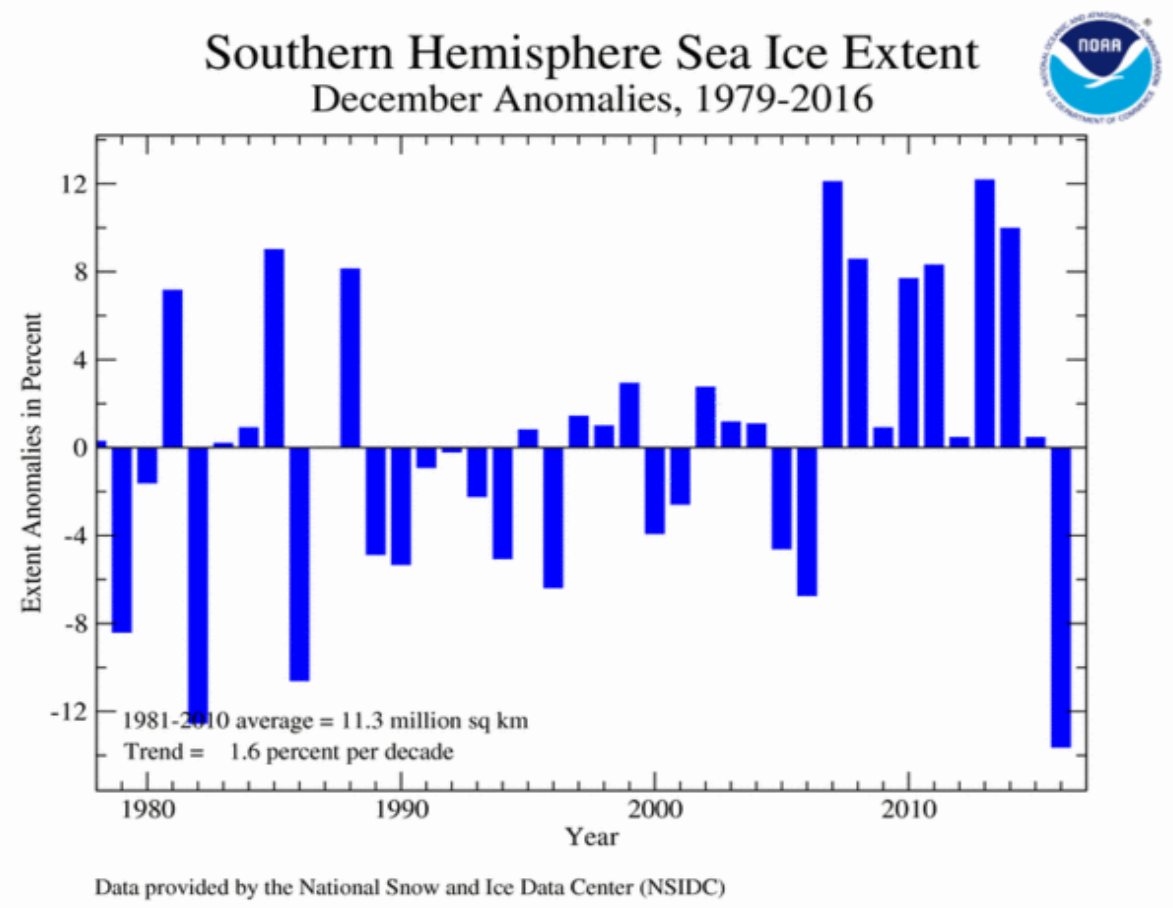

Fig 3 Southern hemisphere sea ice anomalies [7].

area of Antarctica shown in Figs. 4 and 5. The puzzling part of the scenario is that the sea ice area increased in 2007. When studying the SNIT theory it has been determined that supernova debris streams impact the planet for a time period of 50 to 100 years, so why did the sea ice area increase in 2007 [1]? The answer is in Fig 1. The debris stream maximum particle impact areas moved to the south and passed over the sea ice area of the Antarctic to begin calving. At the time the calving data were measured for Fig. 1, the debris stream maximum particle impact areas are melting the bottom of the ice shelves on the continent as indicated by the noted calving large blue areas. The motion of SN 1054 debris streams passing over the sea ice area and traveling south is caused by the changing magnetic field of the sun. The expected large calving area of Fig. 1 caused by the eastern terminus of SN 1054 is missing because the sun is not in the correct position to deflect the eastern terminus debris stream into the continental ice cap. The eastern terminus area of impact with a CAM date of June 12th remains fixed in longitude and latitude year after year. As can be seen in Figs. 4 and 5 the eastern terminus particle stream impacts the sea ice and does not cause calving.

\section{Major Antarctic Loss of Sea Ice}

In 2016, another large decrease in sea ice occurred in Fig 3. This melting occurs due to the impacts of SN 1006's and SN 1054's debris streams in the sea ice area that surrounds Antarctica and is shown in Fig. 6. The nicest part of the SNIT theory is future predictions provide proof of the theory. In the near future, blue areas will develop to the right of the green line as indicated by the purple circles shown in Fig. 2 . When the western terminus of SN 1006 appears in the calving area of Antarctica, it will be accompanied by an area of deflection. A purple circle form the eastern terminus for SN 1006 calving will not exist because this debris stream location will not move south toward the continent and will remain external to the lower continental calving area of Fig. 2.

Since the remnant of SN 1006 has a declination of -42 degrees in the southern hemisphere and is more powerful than SN 1054, the rate of melting at the bottom of the ice shelves due to SN 1006 will be much 
SN 1054, SN 1006, \& Nova WZ Sagittae Calving Antarctic Ice Cap-Global Warming \& Moose Die Off 107 Longitude Relationship Deflection Area in Antarctica and USA-WZ Sagittae Impact and Martian Dust Storms

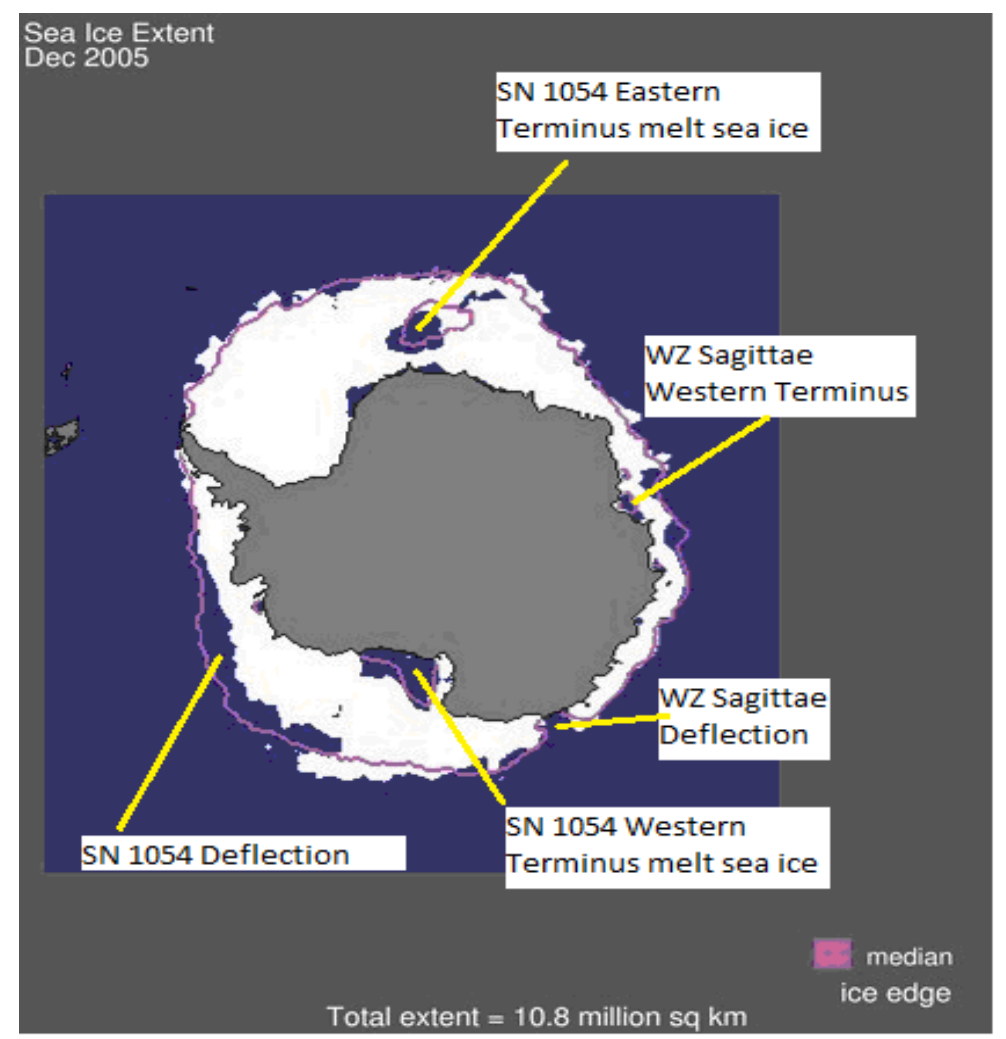

Fig. 4 Antarctic Sea ice melt areas in December 2005 [8].

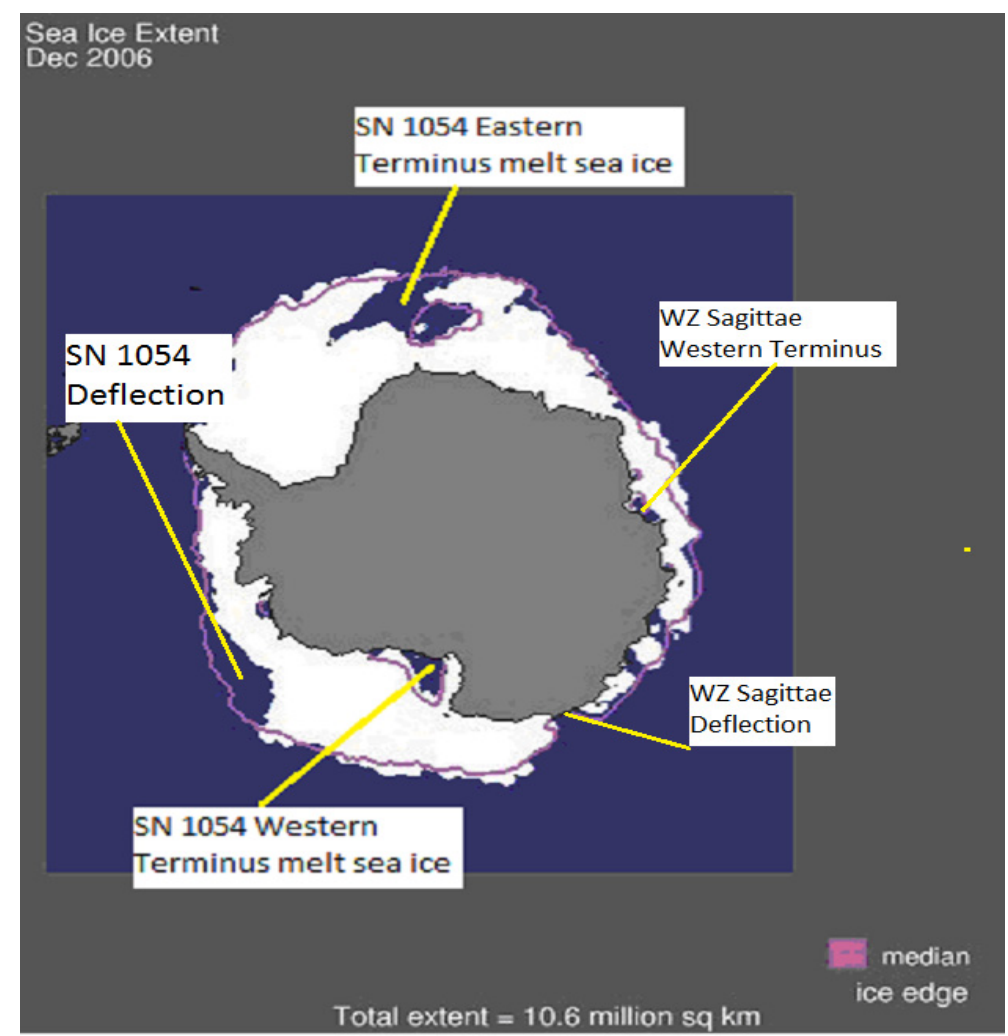

Fig. 5 Antarctic Sea ice melt areas in December 2006 [8]. 
higher than those shown in Fig. 2 for SN 1054 whose remnant has a declination of +20 degrees in the northern hemisphere, but the calving areas may be smaller because of SN 1054 WT and DA movements during the eleven year magnetic cycle of the sun.

\section{Deposit of Particle Stream's Kinetic Energy}

Even though experts will deal with the passage of high velocity particles from exploding stars passing through solids in our future, it is certain when the particle passes through ice and then rock the rock's initial surface of impact will have more heat energy deposited due to the higher density of the rock than the ice. This energy deposited in the rock by SN 1054's debris stream is what causes the bottom ice melting shown in Fig. 1 as green stared blue areas. As the particles penetrate deeper in the Earth, more kinetic energy is converted to thermal energy and high energy magna is produced that has awakened ancient volcanoes. The higher melt rates in Fig. 1 are correctly attributed to volcanoes that have been awakened in Antarctica by the SN 1054 and SN 1006 debris streams.

\section{Slow Motion Picture of Melt Areas}

The eastern termini in Figs. 4 and 5 appear to be fixed in latitude and longitude while the western termini appear to be shrinking or going inland when progressing from year 2005 to 2006 . The motion of the western terminus would agree with the area of calving beginning to form in 2005 as shown in Fig. 1. The area of deflection has become more defined and headed inland in both Figs. 4 and 5. The calving via SN 1054 appears to have started in 2005 and proceed southward for the western terminus and the area of deflection's calving should have started at a later date. The magnetic field of the sun repeats over an eleven-year cycle. If the two calving areas entered the continent near 2005 calving would occur for eleven years and the energy input areas would reemerge at near the same location in the sea ice in 2016. If SN 1006's debris streams contacted the Antarctic sea ice in the same year, 2016, and was moving south, the result would be a composite of two supernovas acting on the Antarctic sea ice in the same year and the results would be as shown in Fig. 6 .

It is interesting that Fig. 6 shows all sea ice melt areas including the volcanoes near shore on western Antarctica. Fig. 6 compares favorably with Figs. 1-5 and it would be expected for the western termini and deflection areas to move south and cause calving on the continent after reducing the area of sea ice melt in a harmonic fashion. The eastern termini tend to remain fixed because the solar magnetic field is not in the path of their particle streams.

The reader can have the practice of explaining the differences in sea ice melt areas between Figs. 6 and 7. I have noted a questionable area in Fig. 7. I will state, at this time, it appears that SN 1054 debris streams continue to withdraw from the continent while SN 1006 debris streams continue to move toward the continent.

\section{Disappearing SN 1054 Eastern Termini Melt Areas}

Figs. 4 and 5 clearly show an area of melt associated with the eastern terminus of SN 1054. When you investigate the monthly figures for the years between 2006 and 2016, the eastern terminus melt area disappears. Where did it go? The debris stream continues to impact the area near June 12th and solar Summer heating occurs in December year after year. Thermal analysis provides the answer. December is like Summer for the south pole sea ice; so, solar heat and kinetic energy from the eastern terminus debris stream in June and December, respectively, help to thin the sea ice, but when the western terminus and deflection debris streams move into the continent to cause calving, their kinetic energies are not added to the clockwise ocean currents that surround the continent and are not convected to the eastern terminus melt area to melt the sea ice in December. 
SN 1054, SN 1006, \& Nova WZ Sagittae Calving Antarctic Ice Cap-Global Warming \& Moose Die Off 109 Longitude Relationship Deflection Area in Antarctica and USA-WZ Sagittae Impact and Martian Dust Storms

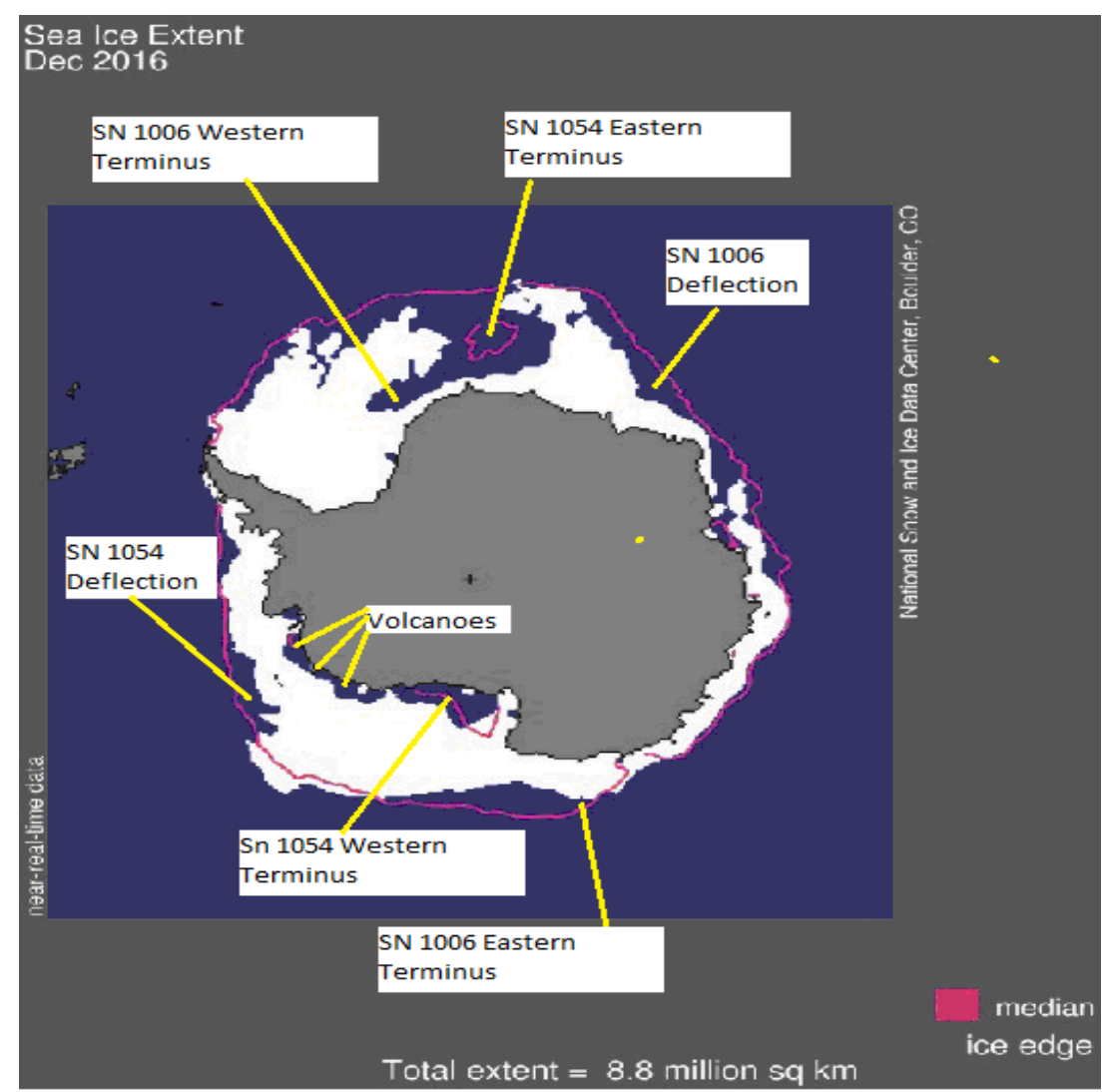

Fig. 6 Antarctic Sea ice melt areas in December 2016 [8].

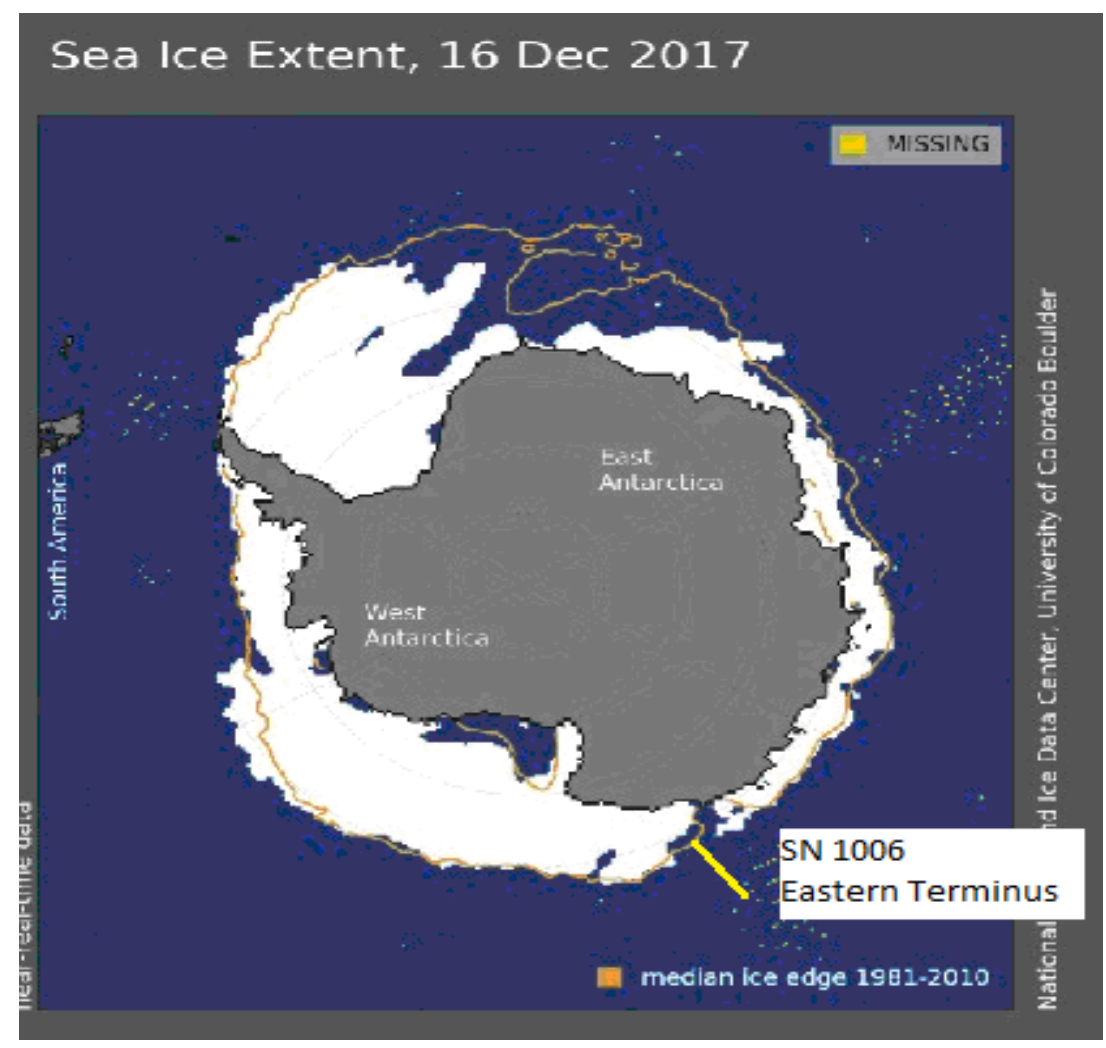

Fig. 7 Antarctic Sea ice melt areas in December 2017. 


\section{Moose Die Off 2006}

The longitude range for the deflection area of the calving of SN 1054 in Fig.1 is 45 to 80 degrees west. The central tine of Satan's pitchfork causes the beginning of the moose die off for the northern USA from Montana to New Hampshire agreeing with the longitude range of the deflection area [9]. Scientists still do not know the reason for the moose die off that is continuing in this area of the USA. This is similar to the Saiga antelope die offs that occur in May 2014 in Asia due to the deflection area of SN 1006 [1].

\section{Central Tine of Satan's Pitchfork Effects USA}

The central tine of SN 1054 termini and deflection areas are located at the same longitudes as the corresponding areas for the southern tine. The longitude and latitude for the eastern terminus area does not change year after year. The longitude for the western terminus and deflection area are also fixed year after year, but the latitude varies in a harmonic motion due to the sun's changing magnetic field during an eleven-year cycle. Two yearly points are noted for impact in the USA, the moose population decline in 2006 and the flu outbreak starting in November 2017 and peaking in January and February of 2018. The period of time from 2006 to 2017 is eleven years matching the solar cycle. In 2009, the USA experienced a swine flu outbreak that occurred near the end of the year [2]. From 2006 to 2009 is three years and from 2009 to 2017 is eight years, totaling to the required eleven years of the solar magnetic cycle. If eleven years are added to the years 2006 and 2009 and then to the results, the series: 2006, 2009, 2017, 2020, 2028, $2031 \ldots$ ad infinitum, denotes the years when unexpected death should hit the USA. The next predicted year for trouble in the USA from SN 1054 is 2020.

Pediatric flu deaths in the USA were 172 for 2017-2018, 171 for 2012-2013, and more than 300 for
2009-2010 [10]. The average number of pediatric flu deaths for a flu season in the USA is 110. The increase in the average number of deaths for 2012-2013 was due to the Dukes effect of the initial impact of SN 1006 and the other two above averages years were due to the deflection area of the central tine of Satan's pitchfork of SN 1054 [2]. In 2009-2010 the flu killed 12,469 Americans [11].

A similar analysis could produce the expected years of increased deaths in Asia due to the central tine of SN 1006 when the location of the future calving due to SN 1006 in Antarctica is known.

SN 1054 also caused a late outbreak of wildfires in the western USA in 2017.

\section{Decrease of Moose Population and Flu Outbreaks Correlation}

The significant declines of the moose population in Minnesota, USA are denoted by the thick black lines in Fig. 8. All the significant declines of the moose population agree with the years of large numbers of flu deaths previously discussed and the year of 2017 is missing in the moose data. The correlation of the years and actions of supernova debris streams allow the realization that the flu not only affects humans but also causes the deaths of the moose in the northern USA. The possibility should be considered that the impacting deflection area debris streams particles weaken the moose causing more wolf kills. The scientists do not know what is causing the new flu strains nor do they know what is causing the decrease in the moose or Saiga antelope population.

\section{Lambert Glacier Calving and Nova $\mathbf{W Z}$ Sagittae}

It is indeed interesting that the calving of the Lambert Glacier near the Amery Ice Shelf, AIS, on the east coast of Antarctica shown at $65 \mathrm{E}$ longitude in Fig. 1 matches the east longitude of the debris stream of Nova WZ Sagittae's western Terminus [1, - Table 6 pg 150], [11]. 


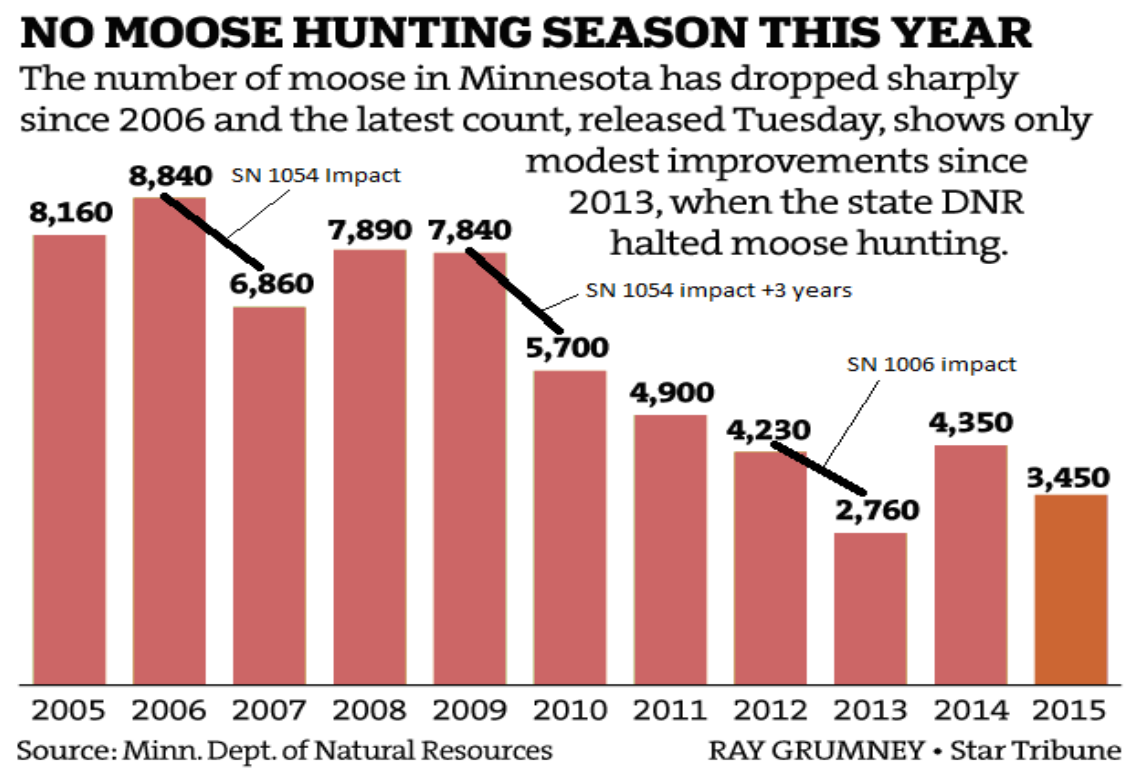

Fig. 8 Moose population decline in Minnesota.

In Figs. 4, 5 and 6, WZ Sagittae's western terminus and deflection area sea ice melts are visible. These melt areas disappear in Figs. 6 and 7 because the debris stream of WZ Sagittae has changed to the cooling or non-heating mode in 2017 [4].

WZ Sagittae is a recurrent nova and has a western terminus particle stream that has eroded the rock surface supporting and under the Lambert Glacier thousands of times over many centuries. The combined effects of the debris streams and the moving ice destroying the rock structure under the Lambert Glacier have caused the Lambert Glacier to be the thickest glacier in the world. The fact that WZ Sagittae is a recurrent nova has caused this stellar scar to exist on our world.

\section{Glacier Velocity 2011}

The Antarctica glacier velocity for the year 2011 is shown in Fig. 9 [12]. The western terminus and deflection area for the particle streams of SN 1054 have high velocity due to the melting ice below the ice stream areas and compare favorably to Fig. 1. A similar comparison exists for the western terminus particle stream of nova WZ Sagittae and the Lambert Glacier. Since this velocity data in Fig. 9 are for 2011 and the initial impact of SN 1006 is in 2011, there will be no evidence of SN 1006 particle streams in Fig. 9.

\section{Martian Dust Storms and Exploding Star's Debris Streams}

This section suggests the solution for NASA proposed problem of how to predict some Martian dust storms. The solution proposes our sun as a mass whose gravity attracts particles expelled from an exploding star, nova or supernova, and focuses the particle stream at specific values of right ascension, RA, as it passes through our solar system. The incoming particle streams will exist at three different right ascension values called zones in our solar system. A dust storm results on Mars when Mars is close to one of the three right ascension zones at the year of the initial wave front of the particle stream. WZ Sagittae is a recurrent nova and it will produce many debris stream wave fronts that will pass through our solar system at different years. Two major outbursts impacted Earth with wave fronts from WZ Sagittae in 1994 and 2018.

Via previous research the times of impact for active particle streams are 2007, 2012, and 2018 for supernova, SN, 1054 known as the Crab Nebula; SN 


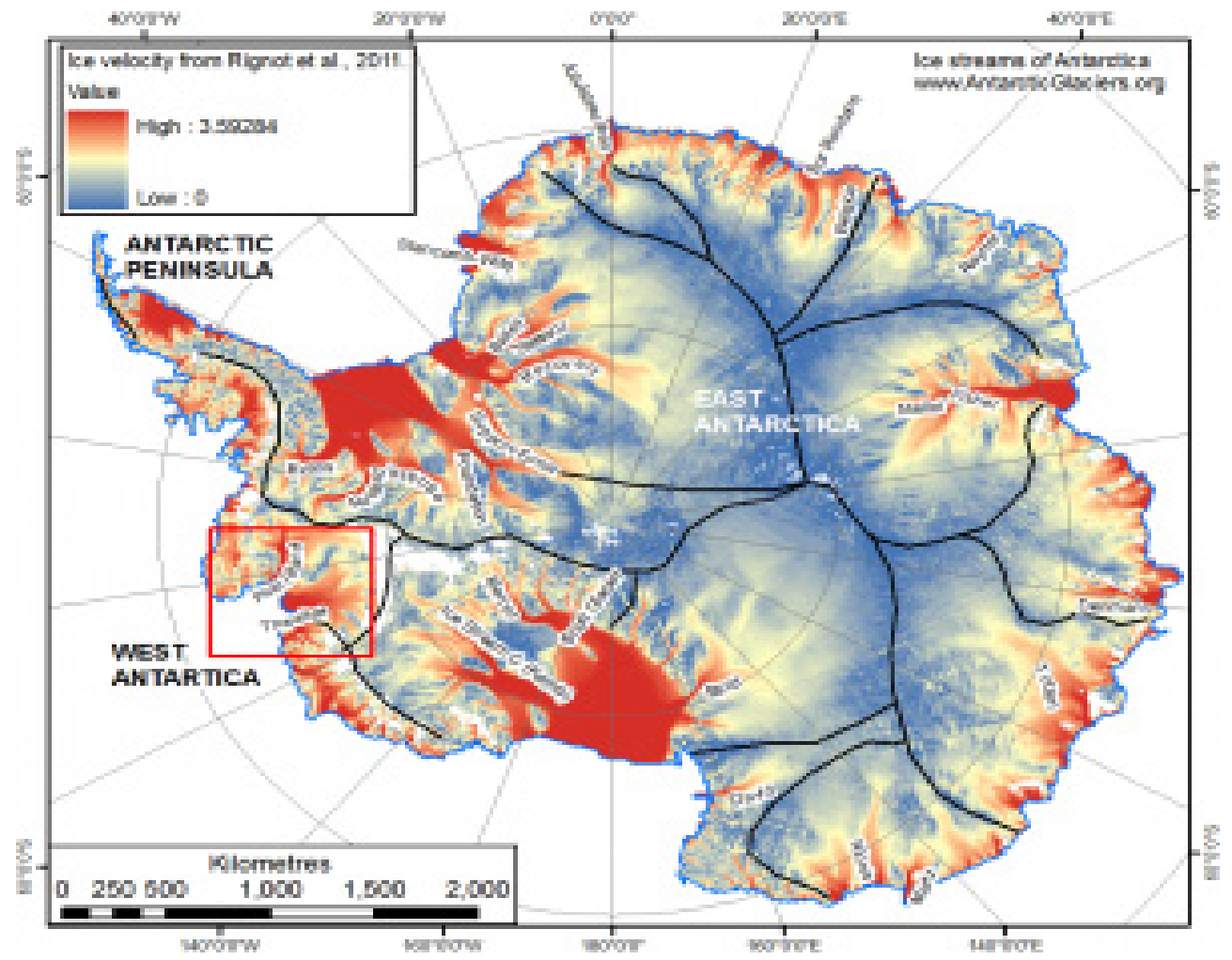

Fig. 9 Glacier velocity 2011.

1006, known as Tyco's supernova; and the current strike of Nova WZ Sagittae, respectively. The planet Mars will be used as a pointer via its right ascension at the time of the dust storm, $\mathrm{M} / \mathrm{D} / \mathrm{Y}$, where the year has been previously noted. If the particle stream is strong enough, dust storms on Mars may occur at the specified right ascension locations at years following the impact year or at different zones at the same year for different particle streams.

\section{Solution for Nasa Dust Storm Correlation}

An Evansville Courier and Press article of June 18, 2018, "It's back: Climate culprit El Nino expected later his year" is a prediction that will occur "big time" at the end of the year 2018 in December. The nova WZ Sagittae has begun a Mega El Nino near June of this year, 2018. A particle stream from the explosion of any star will occur with an initial frontal wave traveling at $88+$ percent the speed of light. When the frontal wave or simply the particle stream passes through our solar system, unusual occurrences result that baffle scientists because exploding stars are not supposed to affect planets in our solar system or the Earth's biosphere. One of the planets in our solar system that is affected in an extreme way by the frontal wave of an exploding star is Mars. Mars a planet with little atmosphere or magnetic field when compared to Earth produced a large dust storm that engulfs the entire planet when it was hit by the frontal wave of Nova WZ Sagittae beginning on June 11, 2018. WZ Sagittae is a recurrent nova and its last major outburst was in 2001. Adding 16.9 years, previously predicted as 18.9 years, to the time of the last major outburst gives a frontal wave that hits Mars in June of 2018 and created a dust storm that turned day into night for NASA's Mars Rover.

The Earth is currently under attack by three exploding stars known as nova and supernova with name/impact year: supernova, SN, 1054/2007, SN 1006/2012 and nova WZ Sagittae/1994 \& 2018. One of the major effects of the impacting debris streams 
of the exploding stars currently active is to add thermal energy to the Earth. The results are melting Earth's ice and increasing sea levels. Fortunately, the melting of the ice via latent heat of fusion absorbs energy without a large increase of Earth's average temperature. The entire process has been called global warming.

Many more effects have been reported for the exploding star's debris streams that have and are impacting our planet. The effects have been reported on internet in the following references [1-3].

The heating mode of the debris stream from Nova WZ Sagittae for the major outburst of 1978 ended in 2016 [4] as was noted by the increase of Arctic sea ice area and the absence of El Nino in December 2017. El Nino will return in 2018 and it will be the beginning of what weather specialists call a Mega El Nino. What has occurred due to WZ Sagittae's previous impacts will reoccur due to the impact of June 2018 and the new debris stream will cause continual heating of our planet maximizing at different time periods of the year at high density particle stream points for the following twenty one years.

The general shape of an incoming debris stream wave front from an exploding star is a cone with an acute angle between the exploding star remnant-sun line and the high particle density center of the cone.

The intensity of the dust storms of Mars is sensitive to Mars' right ascension location with respect to the right ascension of the exploding star as is shown in cases $1 \mathrm{~A}$ and $1 \mathrm{C}$ of the following section. The beginning location of the dust storm is viewed on Mars' surface by the difference of the declination of the remnant with respect to the declination of the planet.

\section{Specifics of the Solution for Martian Dust Storms}

The case number, exploding star name, difference of Mars RA and exploding star RA, and the one RA location (ET, eastern terminus; WT, western terminus;
DA, deflection area) where the planet is impacted by the debris stream are shown as the first line of a specific dust storm.

CASE 1A: Nova WZ Sagittae RA Mars-RA WZ Sagittae $=0$ h $36 \mathrm{~m} 14.2 \mathrm{~s} \mathrm{ET}$

The Mars' dust storm of June 2018 has a June 11th right ascension, RA, for Mars of $20 \mathrm{~h} 43 \mathrm{~m} 50.7 \mathrm{~s}$ and the right ascension for the WZ Sagittae recurrent nova is $20 \mathrm{~h} 7 \mathrm{~m} 36.5 \mathrm{~s}$. The three right ascension zones of maximum particle density for WZ Sagittae are $20 \mathrm{~h} 7$ m $36.5 \mathrm{~s}$ eastern terminus, $8 \mathrm{~h} 7 \mathrm{~m} 36.5 \mathrm{~s}$ western terminus, and $15 \mathrm{~h} 7 \mathrm{~m} 36.5 \mathrm{~s}$ deflection area. The planet Mars for this case is located very close to the debris stream of particles that produce the eastern terminus particle density for Mars and is at one of three locations in Mars' orbit where maximum particle density from nova WZ Sagittae will be encountered by the planet. WZ Sagittae's declination is +17 degrees 42 minutes and Mars declination was -21 degrees 50 minutes.

CASE 1B: Nova WZ Sagittae RA Mars-RA WZ Sagittae $=2$ h $43 \mathrm{~m} 23.5 \mathrm{~s}$ DA

The Mars' dust storm of June 26 to September 4, 2001 shown as Fig. 10 has an initial right ascension, $\mathrm{RA}$, for Mars of $17 \mathrm{~h} 9 \mathrm{~m}$ to $17 \mathrm{~h} 51 \mathrm{~m}$ and the right ascension for the WZ Sagittae recurrent nova is $20 \mathrm{~h} 7$ m 36.5 s. September 4, 2001 was the date the Hubble image showed Mars' surface was totally covered by a dust storm [13]. The three right ascension points of maximum particle density for WZ Sagittae are $20 \mathrm{~h} 7$ m $36.5 \mathrm{~s}$ eastern terminus, $8 \mathrm{~h} 7 \mathrm{~m} 36.5 \mathrm{~s}$ western terminus, and $15 \mathrm{~h} 7 \mathrm{~m} 36.5 \mathrm{~s}$ deflection area. The planet Mars for this case is located close to the debris stream of particles that produce the deflection area zone from WZ Sagittae. WZ Sagittae's declination is +17 degrees 42 minutes and Mars declination was -26 degrees 57 minutes. The temperature rise in the Martian atmosphere during the dust storm was 80 Fahrenheit degrees contributing to the idea that Nova WZ Sagittae is the big contributor to the incoming energy that is causing global warming on Earth. 


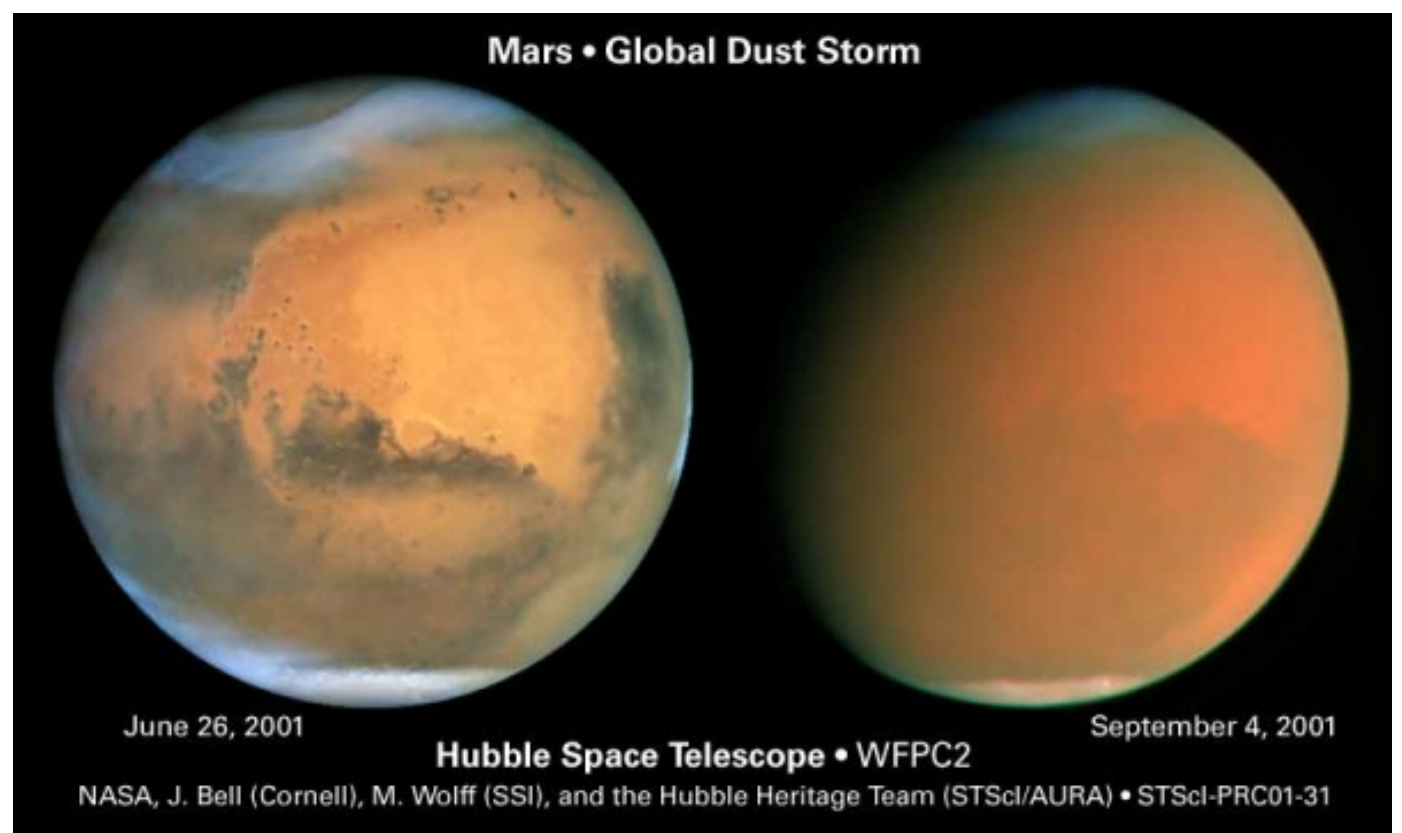

Fig.10 Mars major dust storm in 2001 [15].

CASE 1C: Nova WZ Sagittae RA Mars-RA WZ Sagittae $=0$ h $35 \mathrm{~m} 23.5 \mathrm{~s} \mathrm{WT}$

The Mars' dust storm of August 26 to October 18, 1994 has an initial right ascension, RA, for Mars of 6 $\mathrm{h} 28 \mathrm{~m}$ to $8 \mathrm{~h} 42 \mathrm{~m}$ and the right ascension for the WZ Sagittae recurrent nova is $20 \mathrm{~h} 7 \mathrm{~m} 36.5 \mathrm{~s}$. The three right ascension points of maximum particle density for WZ Sagittae are $20 \mathrm{~h} 7 \mathrm{~m} 36.5 \mathrm{~s}$ eastern terminus, $8 \mathrm{~h}$ $7 \mathrm{~m} 36.5 \mathrm{~s}$ western terminus, and $15 \mathrm{~h} 7 \mathrm{~m} 36.5 \mathrm{~s}$ deflection area. The planet Mars for this case is located close to the debris stream of particles that produce the western terminus zone from WZ Sagittae. WZ Sagittae's declination is +17 degrees 42 minutes and Mars declination was -23 degrees 10 minutes. The temperature rise in the Martian atmosphere during the dust storm was 80 Fahrenheit degrees. This case agrees with the 16.9 years travel time from the WZ Sagittae outburst and Jupiter has a close right ascension and declination values that could deflect the incoming debris stream. The month and day dates of the dust storm were inferred by the outbreak of the black plague on Earth in 1994 and the super outburst of Nova WZ Sagittae in 1978 by adding 16.9 years debris travel time to give a 1994 impact.

CASE 2A: SN 1054 RA Mars-RA SN $1054=-2 \mathrm{~h}$ $1 \mathrm{~m} \mathrm{ET}$

The impact year for the frontal wave of SN 1054 was 2007. The three right ascension points of maximum particle density from SN 1054 are $5 \mathrm{~h} 5 \mathrm{~m}$ eastern terminus, $17 \mathrm{~h} 5 \mathrm{~m}$ western terminus, and $0 \mathrm{~h} 5$ $\mathrm{m}$ deflection area. The right ascension location for Mars on July 20, 2007 when the large dust storm occurred was $3 \mathrm{~h} 4 \mathrm{~m}$. This dust storm will not be a severe as the one Mars is currently experiencing, case 1A because Mars is located $2 \mathrm{~h} 1 \mathrm{~m}$ from the eastern maximum particle density point. SN 1054's declination is +22 degrees 1 minute and Mars declination was +16 degrees. The intensity of debris particles should increase due to the close match of declination for Mars and the remnant of SN 1054.

CASE 2B: SN 1054 RA Mars-RA SN $1054=+3$ h $24 \mathrm{~m} \mathrm{ET}$

The impact year for the frontal wave of SN 1054 was 2007 and a local dust storm occurred on Mars on May 18, 2008 at the northern polar cap. The three right ascension points of maximum particle density from SN 1054 are 5 h 5 m eastern terminus, 17 h $5 \mathrm{~m}$ 
western terminus, and $0 \mathrm{~h} 5 \mathrm{~m}$ deflection area. The right ascension location for Mars on May 18, 2008 when the large regional dust storm occurred was $8 \mathrm{~h}$ $29 \mathrm{~m}$. This dust storm will not be a severe as the one Mars is currently experiencing, case $1 \mathrm{~A}$, because Mars is located $2 \mathrm{~h} 54 \mathrm{~m}$ from the eastern maximum particle density point. SN 1054's declination is +22 degrees 1 minute and Mars declination was +20 degrees 41 minutes that allows a high density side of the cone to cause the dust storm at the north pole on Mars.

CASE 3A: SN 1006 RA Mars-RA SN $1006=+3$ h $6 \mathrm{~m} \mathrm{ET}$

The impact year for the frontal wave of SN 1006 was 2012. The three right ascension points of maximum particle density for SN 1006 after correction are $14 \mathrm{~h} 32 \mathrm{~m}$ eastern terminus, $2 \mathrm{~h} 32 \mathrm{~m}$ western terminus, and $9 \mathrm{~h} 32 \mathrm{~m}$ deflection area. The right ascension location for Mars on November 10, 2012 when the southern hemisphere dust storm occurred was $17 \mathrm{~h} 38 \mathrm{~m}$ being near to the eastern terminus debris stream for SN 1006. SN 1006's declination is -42 degrees and Mars declination was -24.5 degrees. This dust storm was a regional storm in the southern hemisphere of Mars and it is important that the declination of the remnant of the supernova is south of the declination for the planet.

There are numerous cases shown here for dust storms on Mars caused by exploding star debris stream impact. There have been many more dust storms on Mars and Nova WZ Sagittae being a recurrent nova has produced many more outbursts a few of which are shown in Fig. 20 of Ref. [3] and as a result will produce many more dust storms on Mars.

The RA values for the three active exploding stars and their debris streams are shown in Table 1 with the novas S CNC and V606 Aquilae added. The Eastern terminus is defined by the RA of the exploding star. The western terminus is 12 hours from the eastern terminus and seven hours is added the western terminus clockwise to determine the value of the right ascension of the deflection area. The resulting right ascension values for the ET, WT, and DA for the exploding stars are shown in Table 1. The method of correlation assumes the planet with the dust storm is located near one of the three debris stream zones for the exploding star.

A listing of eleven Mars dust storms between 2007 and 2017 follows. The http line is the internet address where the month, day, and year of the Mars dust storm can be found. The lines following the internet location line contain the exploding star (origin of the storm), the nearest debris zone (ET, WT, or DA) match in Table 1 to Mars' right ascension, Mars' right ascension at the date of the storm, Mars' declination at the storm date, and storm date. If the line is followed by the letter E, there is a corresponding Earth dust storm attributed to the exploding star debris stream. If the Mars dust storm is one of the cases mentioned above, the case number and letter are included before the date.

Table 1 Debris stream locations and impact years for exploding stars.

\begin{tabular}{lllll}
\hline & $\begin{array}{l}\text { Eastern terminus, ET } \\
\text { Right ascension }\end{array}$ & $\begin{array}{l}\text { Western terminus, WT } \\
\text { Right ascension }\end{array}$ & $\begin{array}{l}\text { Deflection area, DA } \\
\text { Right ascension }\end{array}$ & $\begin{array}{l}\text { Impact year, IY } \\
\text { Date AD }\end{array}$ \\
\hline WZ SAG & $20 \mathrm{~h} 7 \mathrm{~min} 36.5 \mathrm{sec}$ & $8 \mathrm{~h} 7 \mathrm{~min} 36.5 \mathrm{sec}$ & $15 \mathrm{~h} 7 \mathrm{~min} \mathrm{36.5} \mathrm{sec}$ & 1994,2018 \\
SN 1006 & $14 \mathrm{~h} 32 \mathrm{~min}$ & $2 \mathrm{~h} 32 \mathrm{~min}$ & $9 \mathrm{~h} 32 \mathrm{~min}$ & $2012 \mathrm{AD}$ \\
SN 1054 & $5 \mathrm{~h} 5 \mathrm{~min}$ & $17 \mathrm{~h} 5 \mathrm{~min}$ & $0 \mathrm{~h} 5 \mathrm{~min}$ & $2005 \mathrm{AD}$ \\
S Cnc & $8 \mathrm{~h} 43 \mathrm{~min}$ & $20 \mathrm{~h} 43 \mathrm{~min}$ & $3 \mathrm{~h} 43 \mathrm{~min}$ & $1975 \mathrm{AD}$ \\
V606Aq & $19 \mathrm{~h} 20 \mathrm{~min}$ & $7 \mathrm{~h} 20 \mathrm{~min}$ & $14 \mathrm{~h} 20 \mathrm{~min}$ & $1992 \pm 10$ \\
\hline
\end{tabular}


Beginning of eleven Mars dust storms

https://www.space.com/4101-nasa-fears-dust-storm -doom-mars-rovers.html

1. SN 1054, DA-Mars RA 1 h 47 min Dec. 9 degrees 28 min June 22, $2007|\Delta R A|=1.7 \mathrm{~h}$

The above Mars dust storm suggests the Moose kill of 2007 was near the month of June.

2. SN 1054, ET -- Mars RA 2h 56min Dec 15 degrees 21 min July 17, $2007|\Delta R A|=2.15 \mathrm{~h}$

https://www.jpl.nasa.gov/news/news.php?release $=2$ 007-080

3. SN 1054, ET-Mars RA 3 h 4 min Dec. 16 degrees 0 min Case 2A July 20, $2007|\Delta R A|=2.02 \mathrm{~h}$

https://www.jpl.nasa.gov/news/news.php?release $=2$ 008-210

https://www.jpl.nasa.gov/news/news.php?release $=2$ 008-206

4. WZ SAG, DA-Mars RA 15 h 37 min Dec. -19 degrees $36 \min$ Nov. 11, $2008|\Delta \mathrm{RA}|=0.49 \mathrm{~h}$ $2009 \mathrm{E}$ https://apod.nasa.gov/apod/ap100205.html 5. WZ SAG, WT-Mars RA 8 h 42 min Dec. 22 degrees 54 min Feb. 5, $2010|\Delta \mathrm{RA}|=0.575 \mathrm{~h}$ 2011

http://www.orlandosentinel.com/news/space/go-forlaunch/os-mars-dust-storm-olympus-mons-20180705story.html

6. SN 1006, DA-Mars RA 11 h $58 \mathrm{~m}$ Dec. 0 degrees 37 min July 2, $2012|\Delta \mathrm{RA}|=2.43 \mathrm{~h}$

http://earthsky.org/space/nasa-tracking-a-brewing-d ust-storm-on-desert-world-mars

7. SN 1006, ET-Mars RA 18 h 4 m Dec. 24 degrees $33 \mathrm{~min}$ Case $3 \mathrm{~A}$ Nov. 18, $2012|\Delta \mathrm{RA}|=3.57 \mathrm{~h}$

https://www.bing.com/videos/search?q=2013+janua ry + mars + dust + storm \&qpvt $=2013+$ january + mars + dust + storm\&view $=$ detail\&mid $=03527523$ E4B18989B370 03527523E4B18989B370\&\&FORM=VRDGAR

8. WZ SAG, ET-Mars RA 21 h 0 min Dec. 18 degrees 15 min Jan. 10, $2013 \mathrm{E}|\Delta \mathrm{RA}|=0.875 \mathrm{~h}$
2014

2015

$2016 \mathrm{E}$

http://earthsky.org/space/mars-regional-dust-storms -march-2017

9. SN 1054, DA-Northern Mars RA $0 \mathrm{~h} 58 \mathrm{~min}$ Dec. 6 degrees 3 min Feb. 18, $2017|\Delta R A|=0.82 \mathrm{~h}$

10. SN 1054, DA-Southern Mars RA 1 h $41 \mathrm{~m} \mathrm{Dec.}$ 10 degrees 36 min March 6, $2017|\Delta R A|=1.6 \mathrm{~h}$

Swine Flu outbreak in USA 2017.

https:/www.theverge.com/2018/6/11/17449492/nas a-opportunity-rover-mars-dust-storm-solar-panels

11. WZ SAG, ET-Mars RA 20 h $31 \mathrm{~m}$ to $20 \mathrm{~h} 20 \mathrm{~m}$ May 30 to Aug. 6, $2018 \mathrm{E}|\Delta \mathrm{RA}|=0.39 \mathrm{~h}$

Dust storms in central Asia July 25, 2018 and a major melt near WZ Sagittae weastern terminus sea ice June 2018.

The average value for the absolute value of the difference of RAs, $|\Delta R A|$, between Table 1 value and the date value determined by date in the list of mars dust storms, $|\Delta \mathrm{RA}|$, for the eleven cases listed above is 1.44 hours. The exactness of this method is questionable because the RA width of the dust storm is unknown, but the correct RAs appearing for the supernovas at the predicted year of impact is gratifying (case 1, $2 \& 3$ for SN 1054 year 2007, case $6 \& 7$ for SN 1006 year 2012, and cases $9 \& 10$ SN 1054 year 2017).

Since the WZ Sagittae impact beginning in May 2018 is due to the major outburst of 2001, the ice melts at 115 west longitude, northern Canada, should be unusually large in the summer of 2019 as noted by January 20th debris stream ice thinning of the ET shown in Fig. 10a and Table 6 of reference [1]. Next summer in 2019 the WZ Sagittae WT and DA longitudes in the Arctic should also show large ice melts. The next large melts in the Antarctic should occur at 170 degrees east longitude and 115 degrees west longitude. 


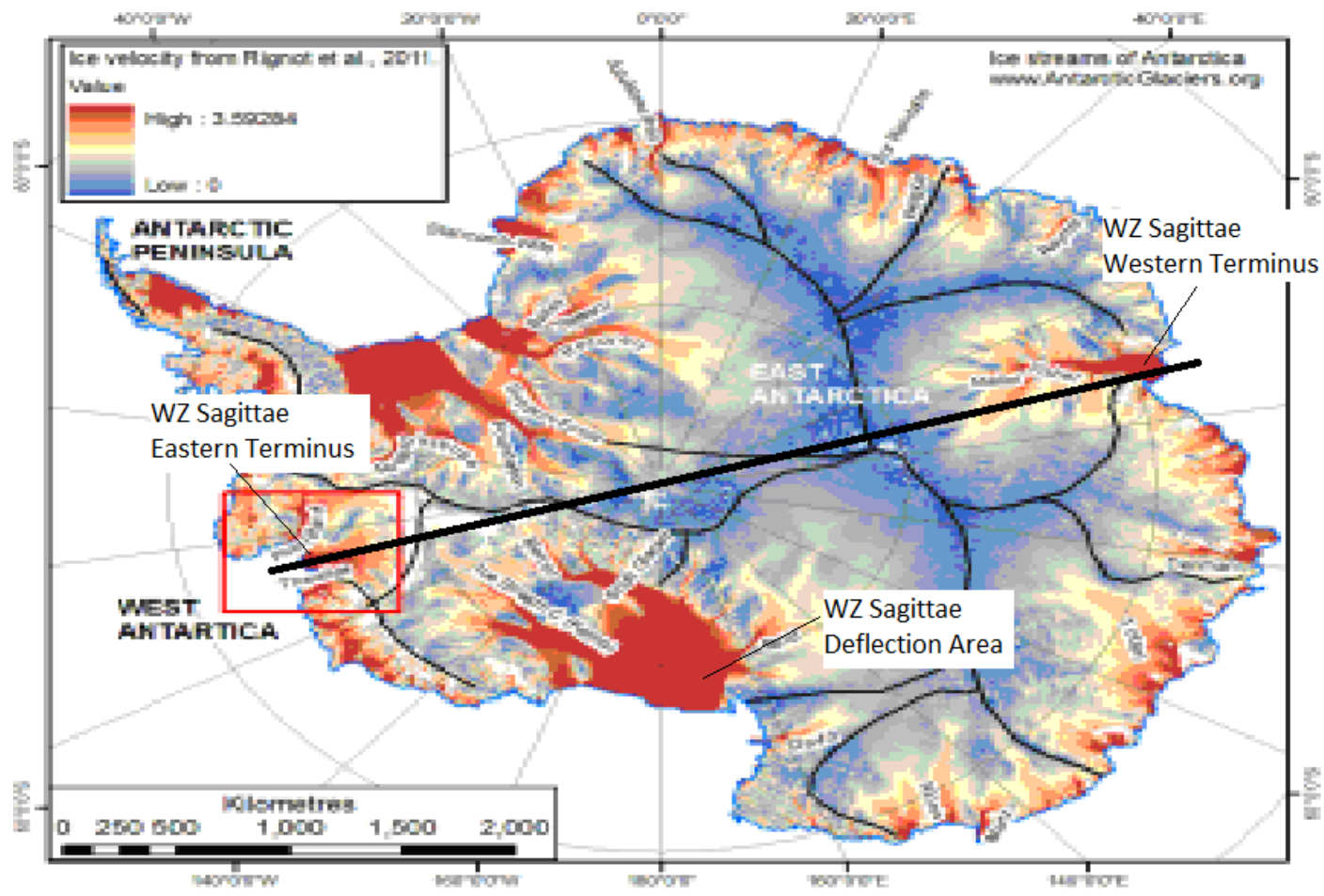

Fig. 10a Deflection area predicts large sea ice melt Barents sea May 30, 2018 [14].

A listing of four Earth dust storms connected to exploding star debris streams follows. The calculation to show the longitudinal predicted location on Earth is shown for clarification.

https://en.wikipedia.org/wiki/2009_Australian_dust storm

1. SAG, DA-Australia Sept. 23, 2009 DOY 266-DOY $201=65$ degrees east from July 20 WT of WZ SAG (Green Line in Australia Fig. 11130 degrees east longitude)

https://www.bing.com/videos/search?q=2013+janua ry + mars + dust + storm\&qpvt $=2013+$ january + mars + dust + storm\&FORM=VDRE

2. SN 1006, ET-Onslow, Western Australia Jan. 11, 2013 DOY 306 to DOY 365 to DOY $11=70$ degrees west (Orange Line Fig. 1197 degrees east longitude)

http://www.esa.int/spaceinimages/Images/2016/07/

Dust_storm_over_the_Red_Sea

3. WZ SAG, DA-Red Sea July, 272016 DOY 20-DOY $201=7$ degrees east from July $20 \mathrm{WT}$ of WZ Sag (Yellow Line Fig. 1172 degrees east longitude)

https://watchers.news/2018/07/27/massive-sandstor m-engulf-golmud-qinghai-province-china/

4. WZ SAG, DA-Golmud, North Western China July 25, 2018 DOY 208-DOY $201=7$ degrees east from July 20 WT of WZ Sag (Blue Line Fig. 1172 degrees east longitude)

The acceptance of an earth dust storm being connected to a particular exploding star depends on the accuracy of the dust storm location using the CAM date of a western or eastern terminus location in conjunction of the one longitude degree per day rule and the understanding of the motion of the maximum particle density point between the two termini.

In Fig. 11, the long horizontal black lines indicate the longitudinal range of the focal point of high particle densities for probable dust storms and they are associated with exploding stars by CAM date and earth longitudinal location. Even though the calculation delivers a point, remember the debris stream covers an area and the variation in degrees may 


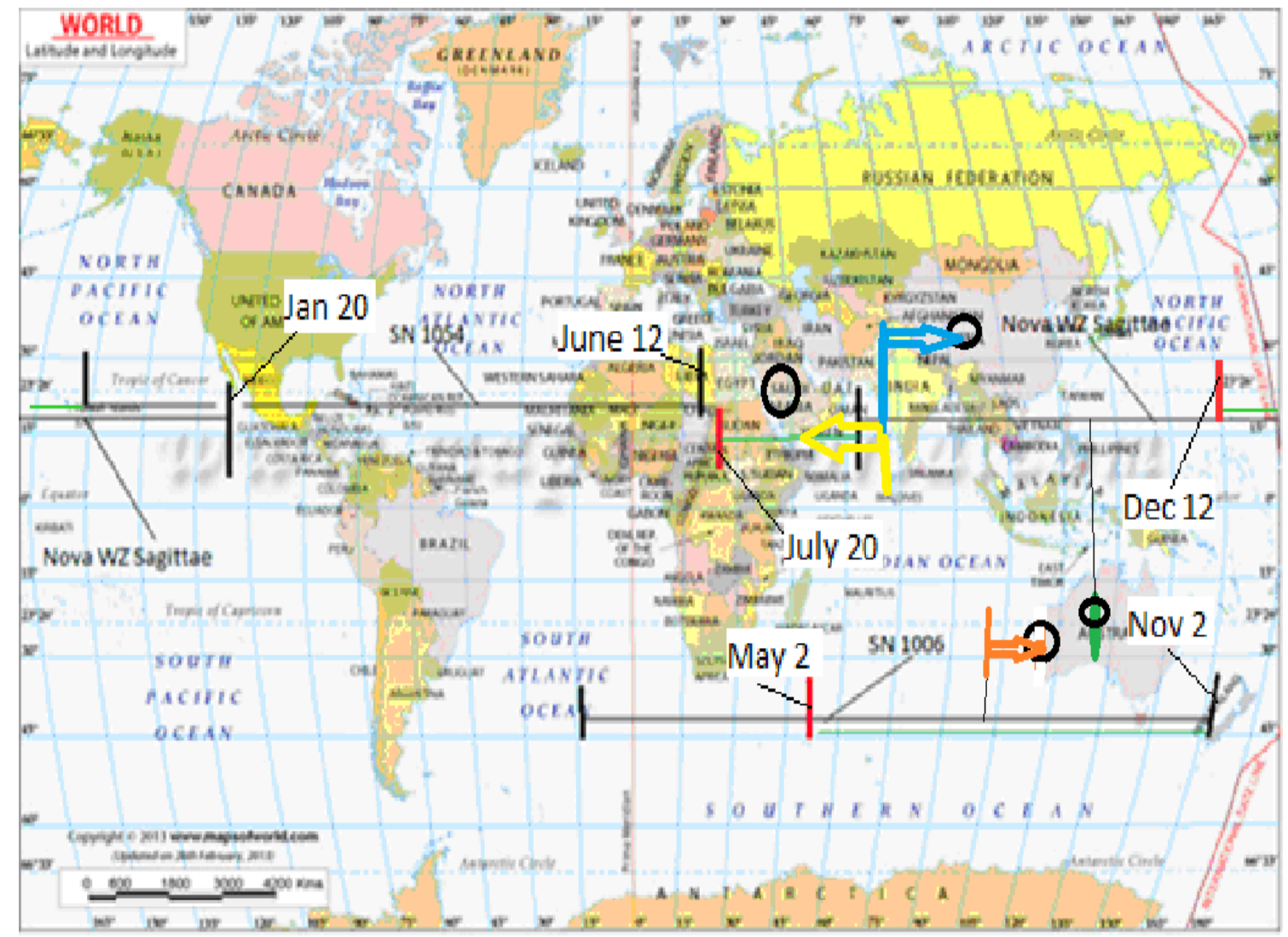

Fig. 11 Certification of earth dust storms with exploding stars.

not be an error (Blue Line to black circle shown by blue arrow, Yellow Line to black circle near Red Sea shown by yellow arrow, and Orange Line to black circle shown by orange arrow).

A listing of earth dust storms not connected to exploding star debris streams are:

https://www.fastcompany.com/1766393/science-be hind-epic-arizona-dust-storm

Arizona July 5, 2011

https://www.nbcnews.com/news/weather/toweringdust-storm-smothers-phoenix-area-n165466

Phoenix July 25, 2014

https://www.bing.com/search?q=Dust+Storm+Phoe nix $+2015 \&$ FORM $=$ HDRSC 1

Phoenix Aug 11, 2015

https://www.azcentral.com/story/news/local/phoeni x-weather/2018/07/05/dust-storm-rolls-into-parts-vall ey/761547002/

Phoenix July 5, 2018
High temperature reaches 115 degrees

https://www.azcentral.com/story/news/local/mesa-b reaking/2018/08/01/dust-storm-advisory-issued-partsphoenix-area/886930002/

Phoenix Aug 3, 2018

All dust storms on Mars are correlated with an exploding star and debris zone for an incoming wave front formed by the explosion. Impact years of the two supernovas, 2007 and 2012, are also correlated by debris zones.

The final proof of the SNIT theory is to realize two planets may be in the same debris zone or stream at near the same time and they should be near a line pointing toward the exploding remnant and our Sun. This occurs with Mars and Earth when they are in opposition in July 2018. Mars has a dust storm that began in June and is continuing to date July 31 st indicating the dust storm has width in the RA direction. The RA location for the WZ Sagittae 


\section{Longitude Relationship Deflection Area in Antarctica and USA-WZ Sagittae Impact and Martian Dust Storms}

remnant is $20 \mathrm{~h} 7 \mathrm{~m} 36.5 \mathrm{~s}$ and the RA location for Mars was $20 \mathrm{~h}$ and $49 \mathrm{~m}$ on July the 6th. On July 27 Mars was in opposition with Earth meaning both planets were in the zone of the debris stream that began impacting our solar system in June of 2018. The large dust storm In Golmud, China of July 25th being focused by earth's magnetic field gives us two dust storms occurring at the same time on the two different planets caused by one visible explosion in the year 2001 of Nova WZ Sagitte. Q. E. D.

The recent impact of Nova WZ Sagittae beginning in May of 2018 was documented by the sudden change to summer temperatures in the spring in Indiana. The drought is also increasing in the USA and is beginning to produce a lower water level two feet below normal in the Kentucky Lake. The wildfires of the western USA have started in July and these can be caused by Nova WZ Sagittae using the Dukes effect indicating a strong Nova WZ Sagittae impact.

\section{Conclusions}

The flow rate of fresh water into the Antarctic Ocean will not increase by a large amount in the next few years due to the energy addition of the particle streams of SN 1006. SN 1006 impacted the planet in 2012 and hit the sea ice around Antarctica in 2016 as can be seen in Fig. 3. The cycle of the sun's magnetic field being eleven years and the fact that the debris streams of SN 1006 have been moving south for five years means the SN 1006 debris streams will start moving north soon and calving due to SN 1006 on the Antarctic continent will be a minimum for this and future solar cycles. The majority of the future calving on the southern continent will be due to nova WZ Sagitte and SN 1054 and the calved areas shown in Fig. 1 will repeat harmonically during the eleven-year solar cycle.

Since all sea ice melt and calving longitudinal locations match the predicted longitudinal locations of high density particle termini for exploding stars that are currently impacting our planet, the SNIT theory is proven correct.

The path thru the Earth and sun magnetic fields for the particles of the southern tine of Satan's pitchfork would be a difficult calculation. If the atomic composition of the debris streams does not change, the large Antarctic sea ice loss experienced in 2016 should reoccur in 2027.

Before the data of Fig. 1 were found, the only connection of SN 1054 debris stream to the moose die off in the northern USA was the year of impact near 2006. Now the longitudinal location has also been verified by the deflection area's noted longitude range.

A study published in the British Journal, Nature, states the ice loss has tripled in Antarctica in the last decade, 2007 to 2017 agreeing with the time span where the SNIT theory implies melting of Antarctic ice by SNs 1054 and 1006 [12].

The retention of heat in the rock layer beneath the Antarctic glacier or ice cap due to penetrating exploding star particles and that heat being released to melt the bottom continental ice that produces warm water that flows into the surrounding ocean is the correct thermo and hydro dynamic model for the major calving areas shown in Figs. 1 and 9. The proposed theory of warm ocean currents flowing 1,000 kilometers, as shown in Fig. 9, under the ice cap to melt the ice cap's bottom ice is incorrect because the weight of the ice is the force that causes the warm water to flow into the ocean. The proposed inflow of warm water is not theoretically possible.

To predict Martian dust storms that can stop the energy of the sun from reaching a solar powered planetary rover you must know the year of impact of the debris stream, the right ascension of the exploding star that gives the three possible orbital locations for the incoming debris stream, and the right ascension value of the planet when the frontal wave will intersect any of the three possible orbital locations for the incoming debris streams.

Since the incoming debris stream from the remnant splits to form the western terminus and deflected area 
streams when the sun is between the remnant and the planet, the strongest dust storms will be at a right ascension location near the eastern terminus' debris stream location.

Numerous dust storms analyzed show accurate right ascension locations for Mars with respect to the remnant's right ascension. The WZ Sagittae dust storms are stronger when compared to the supernova dust storms because WZ Sagittae's remnant is much closer to our solar system than the supernova's remnants.

Facts that produce the severity of the Martian dust storm are the angle of the planet location from the exploding star right ascension remnant-sun line, the distance from our sun to the remnant of the exploding star, the size and power of the exploding star and the time lapse from the year of actual explosion and the year of impact of the initial wave front of the exploding star in our solar system.

The rare event of two different planets experiencing dust storms from the same stellar explosion has been demonstrated.

The SNIT theory predicts past and future occurrences, times and locations, which affect the planet's biosphere. The manmade $\mathrm{CO}_{2}$ theory cannot perform this feat. If man cannot stop the incoming debris particles from exploding stars, man cannot control global weather or killer epidemics.

The fact that two active supernova debris streams produced heating areas that impact the Antarctic sea ice in the same year caused the largest sea ice loss in Antarctica ever recorded is an impressive correlation for the SNIT theory.

The fact that the travel time for the WZ Sagittae debris stream took 16.9 years from the visible 2001 outburst with a remnant 142 light years away gives the constant as 0.119 in the ETA equation [1] and an average velocity of 89.36 percent of light speed. Using these new data causes the GK Persei debris stream to impact Earth between the years 2079 and 2101 AD. If we do a linear interpolation to determine the constant for the distance of GK Persei from Earth, 1500 light-years, the constant equals 0.12164 giving a predicted impact in the year 2083.5 AD. The average velocity for debris streams is faster for closer exploding stars because of some resistance in the ether between stars, but the variation is small. Future researchers could derive average velocity as a function of distance using the points provided by SN 1006 2012 impact and WZ Sagittae 2018 impact and improve the work.

\section{Summary}

Finally in summary, the current strike of WZ Sagittae in 2018 will be the last major outbursting star debris stream impact until GK Persei. Minor outburst from WZ Sagittae will continue yearly because it is a recurrent nova. There may be other impacts if the author has missed the time of a nova or supernova explosions or they were undetected by our astronomers. Currently it is the opinion of the author that the GK Persei explosion's impact may destroy the society of the USA and western France. The article, "The first interstellar asteroid" in April 2018 issue of astronomy indicates the possibility that a number of interstellar asteroids have penetrated Earth causing large exit craters and mega-mass extinctions. The largest exit crater is the Gulf of Mexico and its age is close to the time of the largest mass extinction. For the interstellar asteroid, there is no defense and currently their impact time cannot be predicted, but the world may be able to protect its population from a debris stream impact.

Please send financial support for this research in USA dollars to the Good Shepherd United Methodist Church, 210 W Harrison Street, PO Box 336, Oakland City, In 47660. If you have any questions, the author can be reached by email atwpsokeland@yahoo.com. Good Luck.

\section{References}

[1] Sokeland, W. P. 2017. "Supernova and Nova Explosion's 
SN 1054, SN 1006, \& Nova WZ Sagittae Calving Antarctic Ice Cap-Global Warming \& Moose Die Off

Space Weather: Correlated Megafauna Extinctions and Biosphere Meg-disturbances-Global Warming." Journal of Earth Science and Engineering 2 (2): 136-53. doi:10.17265/2159-581X/2017.03.003.

[2] Sokeland, W. P. 2017. "WZ Sagittae: Space Weather-Global Warming." Journal of Earth Science and Engineering 3: 154-74. doi:10.17625/2159-581X/2017.03.004.

[3] Sokeland, W. P. 2017. "WZ Sagittae, SN 1054, SN 1006 Space Weather-Global Warming" Journal of Earth Science and Engineering 4: 194-216. doi:10.17625/2159-581X/2017.04.002.

[4] Sokeland, W. P. 2018. "Global Warming and Cooling: Friend and Foe to Mankind." Journal of Environmental Risk Assessment and Remediation 2 (2): 8-25.

[5] Sokeland, W. P. 2018. "Snit Theory: Effects of Supernova G11.2-0.3: Current Events SN 1054 \& 1006 and Cholera Environment Entry." Environmental Risk Assessment and Remediation 2 (2): 40-5.
[6] Depoorter, M. A., Bamber, J. L., Griggs, J. A., Lenaerts, J. T. M., Ligtenberg, S. R. M., van den Broeke, M. R., and Moholdt, G. 2013 "Calving Fluxes and Basal Melt Rates of Antarctic Ice Shelves.” Journal of Nature 502 (7469): 89-92.

[7] https://www.ncdc.noaa.gov/snow-and-ice/extent/sea-ice/S $/ 2$.

[8] http://www.climate4you.com/SeaIce.htm\#Southern Hemisphere Monthly Sea Ice.

[9] http://www.climatesignals.org/headlines/events/northernus-moose-decline-2006.

[10] Evansville Courier and Press pg. 7A June 9, 2018.

[11] http://fortune.com/2018/02/10/american-flu-deaths/.

[12] Evansville Courier and Press pg. 9A June 14, 2018.

[13] http://scrippsscholars.ucsd.edu/hafricker/content/icebergcalving-amery-ice-shelf-east-antarctica.

[14] http://www.antarcticglaciers.org/glaciers-and-climate/gla cier-recession/glaciers-and-climate-change/.

[15] http://hubblesite.org/image/1101/news_release/2001-31. 